\title{
FIXED-POINTS OF FINITE GROUP ACTIONS ON CONTRACTIBLE COMPLEXES
}

\author{
BY ROBERT OLIVER ${ }^{1}$ \\ Communicated by Glen E. Bredon, October 1, 1974
}

This is a summary of results obtained while attempting to classify the finite complexes which can be fixed-point sets of cellular actions of a given group on finite contractible $\mathrm{CW}$ complexes. Here, by a cellular action is meant one where the action of any group element takes the interior of any cell to the interior of some other cell, and takes a cell to itself only via the identity map. For groups of prime power order, the question has already been answered by P. A. Smith [4] and Lowell Jones [2]: if $|G|=p^{n}$, a finite complex can be a fixed-point set if and only if it is $\mathbf{Z}_{p}$-acyclic.

The main tools for answering the question for groups not of prime power order are certain functions defined below, which serve as bookkeeping devices for controlling the fixed-point structure of a space with group action. Let $G^{1}$ denote the class of finite groups $G$ with a normal subgroup $P \triangleleft G$ of prime power order such that $G / P$ is cyclic. A resolving function for a finite group $G$ is defined to be a function $\varphi: S(G) \rightarrow Z$ (where $S(G)$ is the set of subgroups) such that:

(1) $\varphi$ is constant on conjugacy classes of subgroups.

(2) $[N(H): H] \mid \varphi(H)$ for all $H \subseteq G$.

(3) $\Sigma_{K \supseteq H} \varphi(K)=0$ for all $H \in \bar{G}^{1}$.

Now define a $G$-resolution of a finite complex $F$ to be any $n$-dimensional $(n-1)$-connected complex $X(n \geqslant 2)$ such that $G$ acts on $X$ with fixed-point set $F$, and such that $H_{n}(X)$ is a projective $Z[G]$-module. To any $G$-resolution $X$ there corresponds a unique resolving function $\varphi$ satisfying

$$
\begin{aligned}
\chi\left(X^{H}\right) & =1+\sum_{K \supseteq H} \varphi(K) \text { for all } 0 \neq H \subseteq G, \\
\sum_{H \subseteq G} \varphi(H) & =0 .
\end{aligned}
$$

AMS (MOS) subject classifications (1970). Primary 55C35; Secondary 57E25.

1 The work described here was done while the author was holding an NSF Graduate Fellowship. 
Conversely, it has been shown that for any $G$ not of prime power order, given an integral resolving function $\varphi$ and a finite complex $F$ with $\chi(F)=$ $\varphi(G)+1$, there is a $G$-resolution $X$ of $F$ which realizes $\varphi$. Letting $m(G)$ denote a generator of $\{\varphi(G): \varphi$ is a resolving function for $G\}$, this proves

THEOREM 1. If $G$ is not of prime power order, then a finite complex $F$ has a G-resolution if and only if $\chi(F) \equiv 1(\bmod m(G))$.

For any $n$-dimensional $G$-resolution $X$ of $F$, an obstruction is defined lying in the projective class group $\widetilde{K}_{0}(Z[G]): \gamma_{G}(F, X)=(-1)^{n}\left[H_{n}(X)\right]$. A subgroup

$$
B(G)=\left\{\gamma_{G}(p t, X): X \text { is a } G \text {-resolution of a point }\right\}
$$

is defined. If $X_{1}$ and $X_{2}$ are two $G$-resolutions of $F$, then $\gamma_{G}\left(F, X_{1}\right)-$ $\gamma_{G}\left(F, X_{2}\right) \in B(G)$, and so there is a well-defined obstruction $\gamma_{G}(F) \in$ $\widetilde{K}_{0}(\mathrm{Z}[G]) / B(G)$, which is zero if and only if $F$ is the fixed-point set of an action of $G$ on some finite contractible complex. It has been shown that $\gamma_{G}(F)$ depends only on $\chi(F)$, and so there is an integer $n_{G}$ such that:

THEOREM 2. For any group $G$ not of prime power order, a finite complex $F$ is the fixed-point set of an action of $G$ on some finite contractible complex if and only if $\chi(X) \equiv 1\left(\bmod n_{G}\right)$.

For the calculation of $m(G)$, the following notation will be used. For $q$ prime, let $G^{q}$ denote the class of all finite groups $G$ with a normal subgroup $H \in G^{1}$ of $q$-power index. Set $G=\bigcup_{q} G^{q}$. Then

THEOREM 3. If $G \in G^{1}$, then $m(G)=0$. If $G \notin G^{1}$, then $m(G)$ is a product of distinct primes (or 1$)$, and $q \mid m(G)$ if and only if $G \in G^{q}$. In particular, $m(G)=1$ if and only if $G \notin G$.

Attempts to calculate $n_{G}$ completely have so far been unsuccessful. The best which has been done is to show that $m(G)\left|n_{G}\right| m(G)^{2}$. In particular, $n_{G}=1$ if and only if $G \notin G$.

Of particular interest is the case of smooth fixed-point free actions on disks. These can be obtained from cellular fixed-point free actions on finite contractible complexes using the methods of [1] or [3]. The above results immediately yield:

THEOREM 4. A finite group $G$ has a smooth fixed-point free action on $a$ (sufficiently high dimensional) disk if and only if $G \notin G$. In particular, any 
nonsolvable group has such an action. A finite abelian group has such an action if and only if it has three or more noncyclic Sylow subgroups.

(Theorem 4 was proved, for solvable groups, in the author's thesis [3] by different methods.)

\section{REFERENCES}

1. E. E. Floyd and R. W. Richardson, An action of a finite group on an n-cell without stationary points, Bull. Amer. Math. Soc. 65 (1959), 73-76. MR 20 \#7276.

2. Lowell Jones, The converse to the fixed-point theorem of P. A. Smith. I, Ann. of Math. (2) 94 (1971), 52-68. MR 45 \#427.

3. R. Oliver, Smooth fixed-point free actions of compact Lie groups on disks, Thesis, Princeton University, Princeton, N. J., 1974.

4. P. A. Smith, Fixed-points of periodic transformations, Amer. Math. Soc. Colloq. Publ., vol. 27, Amer. Math. Soc. Providence, R. I., 1942, pp. 350-373.

DEPARTMENT OF MATHEMATICS, PRINCETON UNIVERSITY, PRINCETON, NEW JERSEY 08540

MATEMATISK INSTITUT, ÅRHUS UNIVERSITET, ÅHUS, DENMARK 